\title{
Comparison of glycemic improvement between intermittent calorie restriction and continuous calorie restriction in diabetic mice
}

Siying Wei ${ }^{1}$, Jingyu Zhao ${ }^{1}$, Meijuan Bai ${ }^{1}$, Chenchen $\mathrm{Li}^{1,2}$, Lingling Zhang ${ }^{1}$ and Yan Chen ${ }^{1,2^{*}}$ (1)

\begin{abstract}
Background: Calorie restriction (CR) has been well proved to be a powerful tool to improve metabolic health associated with aging; and many types of CR have been proposed. Intermittent CR has become a trend in recent years due to its better compliance than continuous CR every day. However, there are few studies that directly compare the interventional activity of intermittent CR vs continuous CR in metabolic disorders such as diabetes.

Methods: In this study, we analyzed two protocols of intermittent CR with the calorie-matched continuous CR in two diabetic mouse models including $d b / d b$ and streptozotocin-treated mice. Intermittent CR was carried out by a fasting-mimicking diet (FMD, with 30\% calorie intake of the control per day) for 2 days or 5 days (i.e., 2-5 or 5-9 regimes followed by free eating for 5 or 9 days respectively).

Results: In the two diabetic mouse models, both intermittent CR and continuous CR significantly reduced fasting blood glucose level and improved insulin sensitivity. However, intermittent CR performed significantly better than continuous CR in improving glycemic control and insulin sensitivity in $d b / d b$ mice. In addition, intermittent CR improved the glucose homeostasis of the $d b / d b$ mice without causing loss of body weight. Analyses with the pancreatic islets reveal that intermittent CR profoundly elevated the number of insulin-positive cells in both diabetic mouse models.

Conclusions: Our study indicated that both intermittent CR and continuous CR can lower fasting blood glucose level in the diabetic mice, while intermittent $C R$ is better than the latter in improving glucose homeostasis in $d b / d b$ mice.
\end{abstract}

Keywords: Diabetes, Intermittent fasting, Fasting-mimicking diet, Calorie restriction, Insulin sensitivity

\section{Introduction}

Weight loss, long considered as a key strategy to ameliorate the progression of type 2 diabetes and calorie restriction (CR), has been widely used to reduce obesity $[1$, $2]$. There are numerous types and regimes of $C R$ used in both animal and human studies. Intermittent $\mathrm{CR}$ or intermittent fasting (IF) has gained a lot of popularity in

\footnotetext{
* Correspondence: ychen3@sibs.ac.cn

${ }^{1}$ CAS Key Laboratory of Nutrition, Metabolism and Food Safety, Shanghai Institute of Nutrition and Health, Shanghai Institutes for Biological Sciences, University of Chinese Academy of Sciences, Chinese Academy of Sciences, 320 Yueyang Rd, Shanghai 200031, China

${ }^{2}$ School of Life Sciences and Technology, Shanghai Tech University, Shanghai 200031, China
}

recent years as it is easily acceptable to patients [3]. Intermittent $\mathrm{CR}$ refers to a dietary pattern of extended time period of fasting with low or no energy intake (e.g., 16-48 h), followed by a period of normal food intake, on a recurring basis [4]. It is believed that intermittent CR can induce a metabolic switch from lipid/cholesterol synthesis and fat storage to mobilization of fat through fatty acid oxidation and fatty acid-derived ketone bodies which have the potential to improve body composition in overweight individuals [5]. In laboratory animals, intermittent $\mathrm{CR}$ has profound beneficial effects on metabolic health, slow down disease processes, and improve age-related disorders including diabetes, cancers, 
cardiovascular disease, and neurodegenerative disorders [4]. The common concept in the field is that CRmediated loss of obesity is the primary cause of glycemic improvement in diabetes. However, it was found that calorie restriction is a significant factor in glycemic control in obese type 2 diabetic patients, in addition to the magnitude of weight loss [6]. In this study, we investigate whether CR-mediated weight loss is associated with glycemic control in diabetic mice.

To increase the compliance of intermittent $C R$ in humans, Dr. Longo's group has proposed the concept of fasting-mimicking diet (FMD) instead of simple fasting [7]. Application of FMD for 4 days every 2 months in middle-aged mice led to a reduction in cancer incidence, extension in lifespan, and decrease in visceral fat [7]. Additionally, intermittent use of FMD improved demyelination in a murine model of experimental autoimmune encephalomyelitis [8]. The combination of FMD with chemotherapy delayed the progression of melanoma and breast cancers via boosting $\mathrm{T}$ cell-mediated cytotoxicity [9]. Recently, a human experiment with 100 healthy subjects indicated that administration of a FMD low in calories, sugar, and protein, but high in unsaturated fat, had favorable effects on body mass index and blood pressure, in addition to improvements in blood levels of fasting glucose, C-reactive protein, insulin-like growth factors-1 (IGF-1), triglycerides, total cholesterol, and low-density lipoprotein cholesterol in subjects at risk for diseases [10]. Lately, FMD was shown to improve glucose homeostasis, promote $\beta$ cell generation, and restore insulin secretion in type 1 and type 2 diabetic mouse models [11], heralding the potential use of the FMD to reverse the pathogenesis of diabetes.

Although a plethora of studies have investigated the interventional activities of different modes of CR, there are few reports that directly compare intermittent $C R$ vs continuous CR in the same study. In this study, we compared the interventional efficacy of intermittent $C R$ vs continuous $\mathrm{CR}$ in both type 1 and type 2 diabetic mouse models. Intriguingly, we found that while both intermittent $\mathrm{CR}$ and continuous $\mathrm{CR}$ could improve glycemic control in the mice, intermittent CR performed better than continuous $\mathrm{CR}$ in certain aspects.

\section{Experimental methods \\ Mouse model}

Four to six-week-old male C57BL/ksJ-db $(d b / d b)$ mice and normal C57BL/6 mice purchased from SLAC (Shanghai, China) were maintained in pathogen-free conditions and kept on a $12 \mathrm{~h}$ light/dark cycle at the Institute for Nutritional Sciences. All mice were fed until they were 10-weeks-old and then weighed at the beginning of the study and randomly allocated to experimental groups. The control group was fed with normal chow with free access to food and water. Two regimes of intermittent CR (2-5 and 5-9) was used with calorie restriction for 2 or 5 days followed by free access to normal chow for 5 days or 9 days respectively. Intermittent CR was administered by a FMD that contained low level of protein and high level of fiber [12]. During the use of FMD, the calorie intake was $30 \%$ of the daily calorie intake of the normal control group. We also included two groups of continuous CR that matched the total calculated calorie intake of the 2-5 and 5-9 intermittent FMD groups (with $\sim 81 \%$ and $\sim 76 \%$ of daily calorie intake of the normal control group respectively). CR group was fed with normal chow as control group. Before the beginning of the experiment, we recorded the average food intake per day per mice for one week. Based on the recorded value, we calculated the amount of FMD (1/3 cal intake per day during the fasting period) and that of continuous $\mathrm{CR}$. In addition, the mice of intermittent FMD and continuous CR groups were caged individually to make sure the food intake was well controlled. The food intake of the mice was carefully recorded to make sure that the calorie intake of intermittent FMD group and continuous calorie restriction (CR) group were strictly controlled throughout the entire period of the experiment (Additional file 1: Figure S1). The animal experiments were conducted in accordance with the guidelines of the Institutional Animal Care and Use Committee of the Institute for Nutritional Sciences, Shanghai Institutes for Biological Sciences (SIBS), Chinese Academy of Sciences (CAS) with an approval number 2010-AN-8. For inducing T1D, male C57BL/6 mice were fasted $4 \mathrm{~h}$ prior to STZ injection $(40 \mathrm{mg} / \mathrm{kg})$. STZ was purchased from Sigma-Aldrich (Cat. No. S0130) and dissolved in sodium citrate buffer $(\mathrm{pH} 4.5$, freshly prepared) at a concentration of $4 \mathrm{mg} / \mathrm{ml}$ immediately prior to use and STZ solution was administered intraperitoneally within $5 \mathrm{~min}$ according to mice weight. Mice were then returned to their cage and $10 \%$ sucrose water was provided to prevent hypoglycemia after STZ injection. Two weeks after STZ injection, all mice became hyperglycemia ( $>10 \mathrm{mmol} / \mathrm{ml}$ ). For T2D, four to six-week-old male C57BL/ksJ-db $(d b / d b)$ mice were purchased from SLAC (Shanghai, China). All mice were fed to become hyperglycemia $(>10 \mathrm{mmol} / \mathrm{ml}$ ) after 2 or 3 weeks before intervention.

\section{Fasting mimicking diet}

The FMD used in this study (Gembynear Nutrition Bar or Zhenbainian in Chinese) was kindly provided by Beijing Winlife Research Institute of Nutrition, Health, Food Science, and Technology (Beijing, China). The nutritional composition of the FMD was provided as previously reported [12]. During the use of FMD, all mice were supplied with fresh food in the morning ( 9:00 
a.m.). The mice usually consumed the supplied food within the first few hours.

\section{Blood glucose and insulin measurement}

All mice were fasted for $6 \mathrm{~h}$ in the morning before blood glucose measurements. Blood glucose was measured through the tail vein by the use of the OneTouch UltraEasy Blood Glucose Monitoring System (Lifescan, Milpitas, CA, USA). Serum insulin levels were determined by a murine enzyme-linked immunosorbent assay kit (Shanghai Enzyme-linked Biotechnology Co., Shanghai, China). Whole blood was withdrawn from the orbital sinus, and plasma was separated by centrifugation at $3400 \mathrm{rpm}$ for $15 \mathrm{~min}$ in EDTA-K2-treated microtubes (Kangjian Medical, China).

\section{Glucose tolerance testing (GTT) and insulin tolerance testing (ITT)}

Mice were caged individually and fasted for $4 \mathrm{~h}$ for ITT in the morning and fasted overnight for GTT. Glucose $(1 \mathrm{~g} / \mathrm{kg})$ or insulin (3 unit $/ \mathrm{kg}$ ) was injected intraperitoneally. Blood glucose levels were measured from $0 \mathrm{~min}$ to 120 min after injection.

\section{Immunofluorescence analysis}

Mice pancreas samples were collected then rinsed in PBS and fixed in 4\% paraformaldehyde overnight before being dehydrated and embedded into paraffin. After that, samples were sectioned into $4 \mu \mathrm{m}$ slices. The immunofluorescence staining was performed as previously described [12]. The following primary antibodies were used: anti-insulin (cat. no. C27C9, Cell Signaling Technology, Danvers, MA, USA), anti-glucagon (cat. no. ab10988, Abcam, Cambridge, UK). The nucleus was stained with Hoechst 33342 (Eugene Oregon, USA). The images were captured by a 40x objective with an LSM 510 confocal microscope (Zeiss, Jena, Germany).

\section{Measurement of serum and hepatic parameters}

Mice were euthanized, and the blood of the mice was immediately collected from mice orbital sinus into EDTA-K2-treated microtubes (Kangjian Medical, China). After that, the microtubes were centrifuged at $3400 \mathrm{rpm}$ for $15 \mathrm{~min}$, and the blood serum was then collected. Except for those for immediate use, all serum samples were stored at $-80^{\circ} \mathrm{C}$. Serum levels of aspartate transaminase (AST) and alanine transaminase (ALT) were determined by an AST/ALT determination kit (ShenSuo UNF, China). Serum levels of triglycerides (TG) and total cholesterol (TC) were determined by colorimetric methods with the corresponding kits (ShenSuo UNF, China). All of these assays were performed as previously described [12].

\section{Antibodies and immunoblotting}

The antibodies against total AKT and phosphorylated AKT were from Cell Signaling Technology (Danvers, MA, USA). The protocols for immunoblotting have been described previously [13].

\section{Statistical analysis}

All data were expressed as the mean \pm SEM. Significant differences were assessed by one-way ANOVA followed by the Student-Newman-Keuls test where appropriate or by two-tailed Student's t test. Statistical tests were performed using Microsoft Excel (Microsoft, Redmond, WA, USA), R v3.3.2, or Prism6 (GraphPad Software, La Jolla, CA, USA) where appropriate.

\section{Results}

Comparison of intermittent CR and continuous CR in $d b$ / $d b$ mice

We first compared the interventional effect of 2-5 regime of intermittent CR vs continuous CR in $d b / d b$ mice that had severe type 2 diabetes due to extreme obesity caused by deficiency of leptin receptors. For implementation of intermittent $\mathrm{CR}$, we used a fasting-mimicking diet (FMD) that is vegetable-based, and lower in protein and carbohydrates compared to normal chow [12]. The $d b / d b$ mice were divided into three groups (Fig. 1a). The control group had ad libitum (AL) access to normal chow. The intermittent FMD group was exposed to FMD for 2 days (with $1 / 3$ cal intake of the control) and ad libitum diet for 5 days per week. The matched continuous CR group had normal chow with total energy intake equal to the calculated intermittent FMD group ( $81 \%$ energy intake of the control group per day). During the 13 weeks of intervention, the body weight of the three groups of mice was not significantly different from each other (Fig. 1b). The elevated fasting blood glucose level in $d b / d b$ mice was significantly reduced by both intermittent FMD and continuous CR (Fig. 1c). Analysis with the area under the curve revealed that the cumulative fasting blood glucose level in the intermittent FMD group was lower than that of continuous CR group (Fig. 1c). Both GTT and ITT were performed at the end of the experiment with the animals. The glucose tolerance of the mice was significantly improved by intermittent FMD, but not by continuous CR (Fig. 1d). Insulin sensitivity was improved by both intermittent FMD and continuous $\mathrm{CR}$, while intermittent FMD performed better than continuous CR (Fig. 1E). In addition, the blood insulin level was significantly elevated by both intermittent FMD and continuous CR (Fig. 1f). However, the serum levels of total cholesterol (TC) and triglyceride (TG) were not altered by either type of CR (Fig. $1 \mathrm{~g}$ and $\mathrm{h}$ ). Only continuous CR reduced the serum level of ALT 


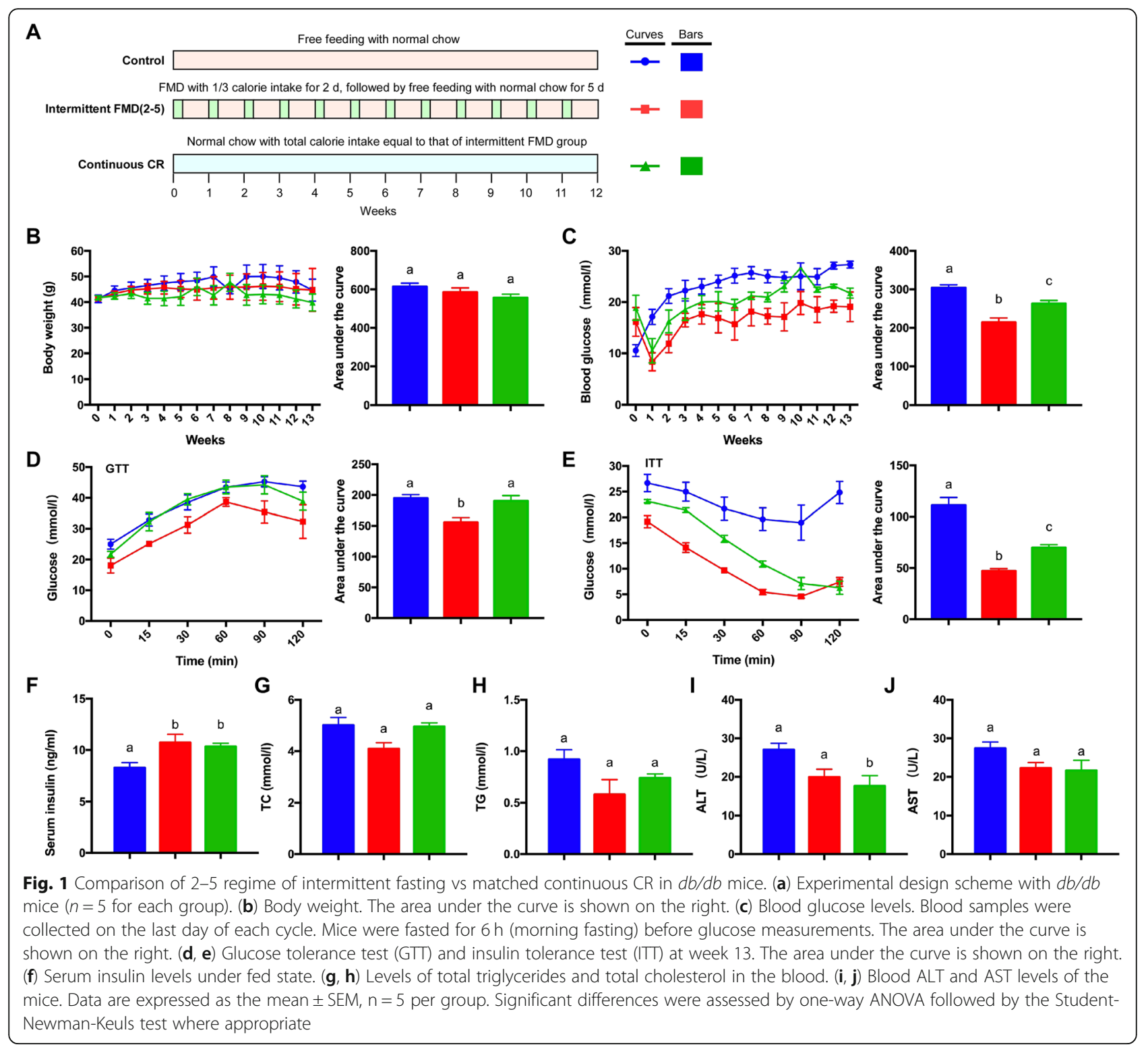

(Fig. 1i). However, the serum level of AST was not altered by either type of fasting (Fig. 1j).

We next analyzed another regime of intermittent FMD together with the calorie-matched continuous CR. The $d b / d b$ mice were divided into three groups (Fig. 2a). The control group had free access to normal chow. The intermittent FMD group was exposed to an alternating dietary pattern that consisted of FMD for 5 days and free access to normal chow for 9 days. The energy intake during FMD days was $1 / 3$ of daily energy intake of the control group. The calorie-matched continuous CR group had normal chow with total energy intake equal to the intermittent FMD group ( $76 \%$ of energy intake of the control group per day) (Fig. 2a). During the 12 weeks of intervention, the cumulative body weight of the continuous $\mathrm{CR}$ group was significantly lower than the weight of the other two groups (Fig. 2b), indicating continuous $\mathrm{CR}$ is better than intermittent $\mathrm{CR}$ in body weight reduction. The elevated fasting blood glucose level in $d b / d b$ mice was significantly reduced by both intermittent FMD and continuous CR. However, intermittent FMD was better than continuous CR in reducing fasting blood glucose level (Fig. 2c). GTT revealed that both intermittent FMD and continuous CR significantly improved glucose tolerance (Fig. 2d). ITT showed that both intermittent FMD and continuous CR improved insulin sensitivity, while intermittent FMD performed better than continuous $\mathrm{CR}$ in this assay (Fig. 2e). The serum levels of insulin, TC, and TG were not significantly changed among the three groups of mice (Fig. $2 \mathrm{f}$ and $\mathrm{h}$ ). However, the blood ALT level was reduced by both intermittent FMD and continuous CR (Fig. 2i), 


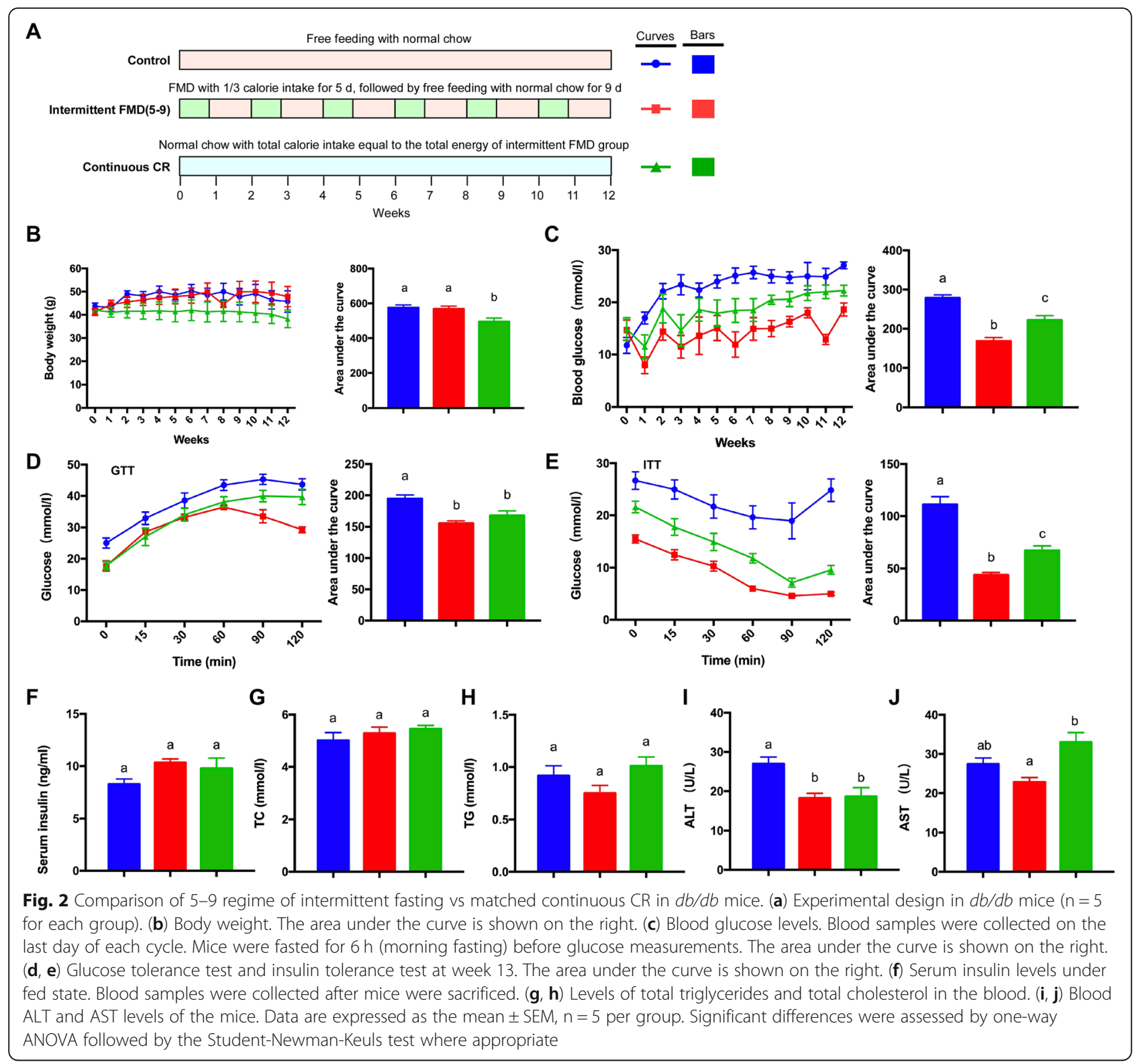

while the blood AST level was not significantly altered by either type of calorie restriction (Fig. 2j).

To rule out the possibility that observed difference in the study between intermittent FMD and continuous CR was caused by the FMD, we analyzed intermittent $C R$ with normal chow. One group of intermittent $C R$ with normal chow was added in the 2-5 regime experiment (Additional file 1: Figure S2A). The cumulative body weight was not altered by intermittent $C R$ with normal chow (Additional file 1: Figure S2B). The decrease of fasting blood glucose level of intermittent CR with normal chow was the same as intermittent FMD, while better than continuous CR (Additional file 1: Figure S1C). In addition, the glucose tolerance and insulin sensitivity of the intermittent $\mathrm{CR}$ with normal chow group were not significantly different from the intermittent FMD group (Additional file 1: Figure S1D and E). Overall, these data indicated that intermittent FMD is identical to intermittent $\mathrm{CR}$ with normal chow to improve glycemic control in the $d b / d b$ mice when using the 2-5 regime of fasting.

\section{Comparison of intermittent fasting and continuous $C R$ on intervention of STZ-induced diabetic mice}

To further explore the interventional effect of intermittent fasting or continuous $\mathrm{CR}$ on diabetes, we analyzed a type 1 diabetes (T1D) mouse model that was generated by STZ injection in C57BL/6 mice. We first analyzed the 2-5 regime of intermittent FMD together with the calorie-matched continuous CR (Fig. 3a). During 13 


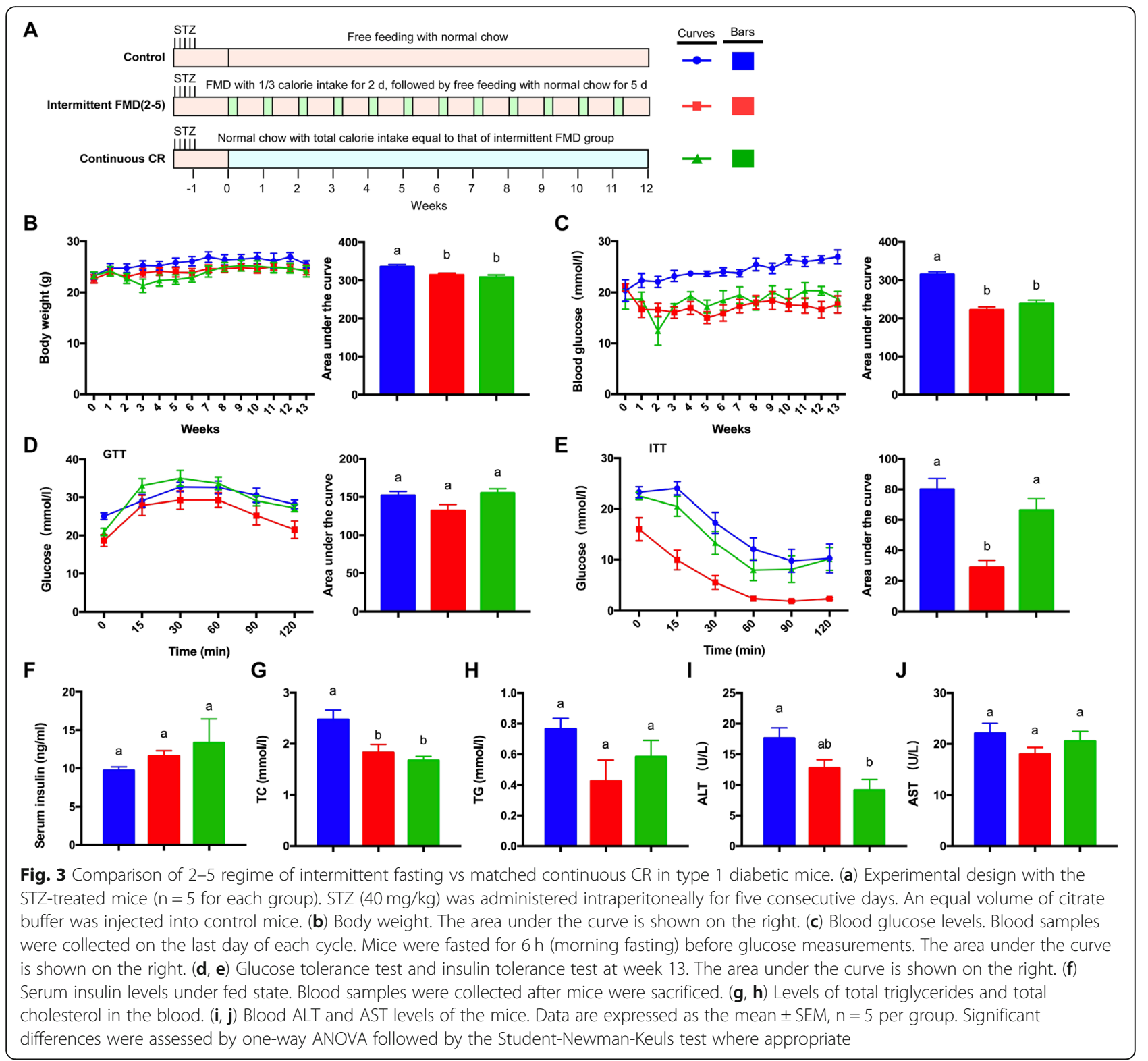

weeks of intervention, the cumulative body weight of the mice was significantly reduced by both intermittent FMD and continuous CR (Fig. 3b). The elevated fasting blood glucose level in the T1DM mice was significantly reduced by both intermittent FMD and continuous CR (Fig. 3c). GTT was not significantly altered by either type of fasting (Fig. 3d). However, ITT revealed that only intermittent FMD could significantly improve insulin sensitivity (Fig. 3e). However, the blood insulin level was not significantly different among the three groups of mice (Fig. 3f). Both intermittent FMD and continuous CR reduced the serum total cholesterol level (Fig. 3g), while the blood triglyceride level was not altered (Fig. 3h). The blood ALT level was significantly decreased by continuous CR (Fig. 3i), while the blood AST level was not changed (Fig. 3j).
We also investigated the 5-9 regime of intermittent FMD with the T1D mice together with the caloriematched continuous CR (Fig. 4a). With this regime, the cumulatively body weight of the mice was significantly reduced by both intermittent FMD and continuous CR (Fig. 4b). The elevated fasting blood glucose level in the T1DM mice was significantly reduced by both intermittent FMD and continuous CR (Fig. 4c). GTT revealed that only intermittent FMD could significantly improve glucose tolerance (Fig. 4d). However, the insulin sensitivity of the mice was improved by both intermittent FMD and continuous CR (Fig. 4e). The blood insulin level was not altered in these mice (Fig. 4f). Only intermittent FMD reduced the total cholesterol and triglyceride levels of the mice (Fig. $4 \mathrm{~g}$ and $\mathrm{h}$ ). The blood levels of ALT and 


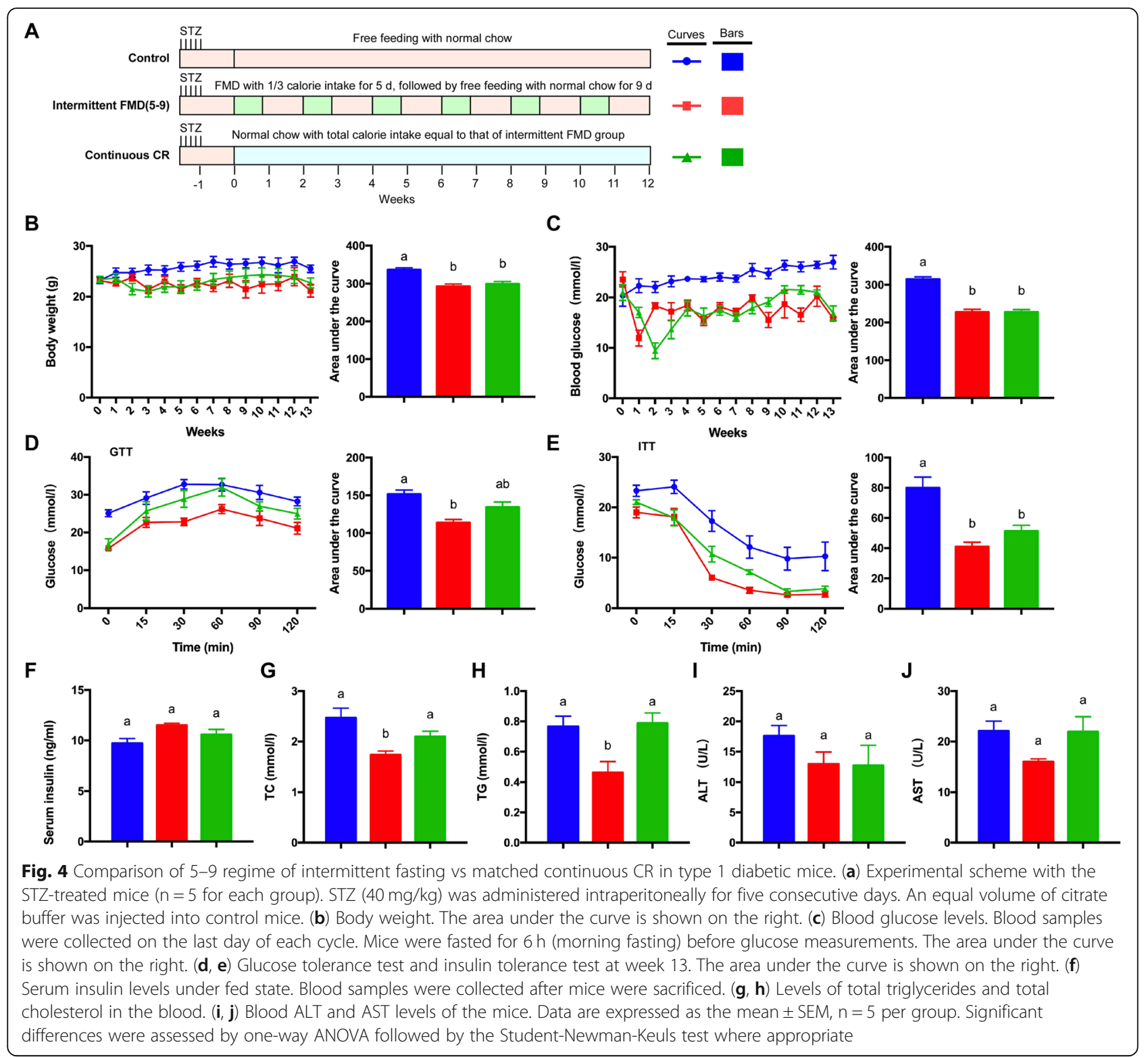

AST was not altered by either type of calorie restriction (Fig. $4 \mathrm{i}$ and $\mathrm{j}$ ).

\section{Summary of the interventional efficacy of intermittent FMD vs continuous CR on both types of diabetes in the mice}

We summarized the animal data of our study (Fig. 5). In $d b / d b$ mice, only continuous CR that matched the 5-9 regime of intermittent FMD significantly reduced the body weight. Both intermittent FMD and continuous CR significantly reduced the fasting blood glucose level. However, intermittent FMD performed better than continuous $\mathrm{CR}$ in this aspect. Both regimes of intermittent FMD significantly improved glucose tolerance, while only continuous CR matching the 5-9 regime of intermittent FMD improved it. In terms of insulin sensitivity, both intermittent FMD and continuous CR improved it, while intermittent FMD performed better than continuous CR. Overall, our data suggests that intermittent FMD has a better effect than continuous $\mathrm{CR}$ on reducing fasting blood glucose level and improving insulin sensitivity in $d b / d b$ mice.

In the T1D mouse model, both intermittent FMD and continuous CR significantly reduced the body weight and the fasting blood glucose level (Fig. 5). The 5-9 regime of intermittent FMD improved glucose tolerance of the mice, while continuous $C R$ had no effect in this aspect. The 2-5 regime of intermittent FMD but not its matched continuous CR improved insulin sensitivity. However, both 5-9 regime of intermittent FMD and the 


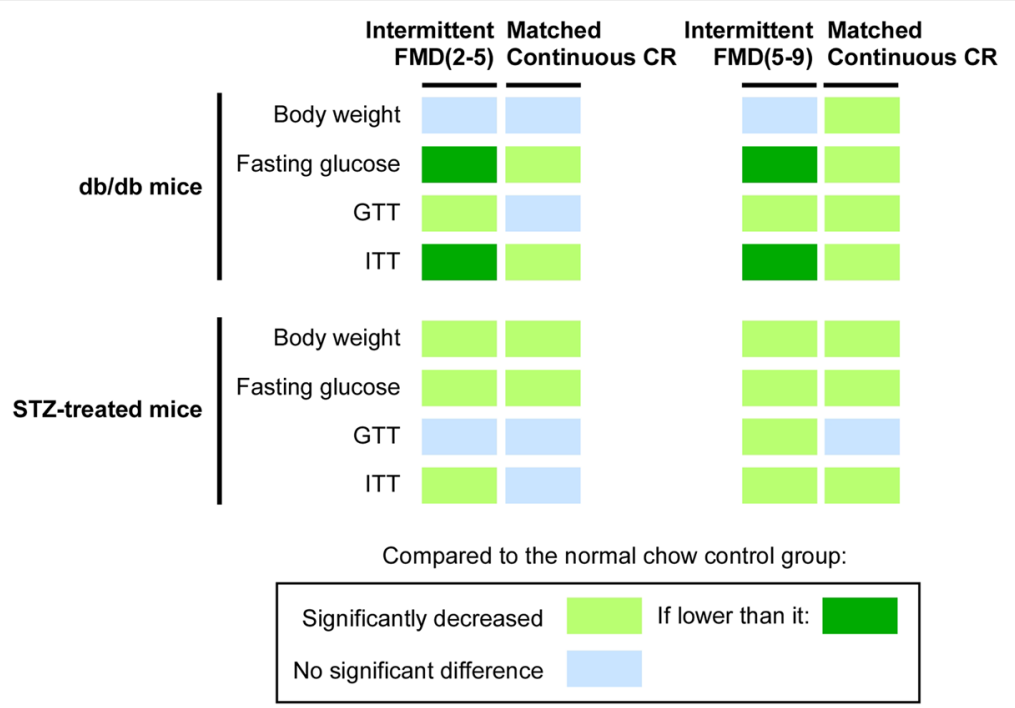

Fig. 5 Summary of different regimes of fasting on glycemic control of different diabetic mouse models. Only the areas under the curves from Figs. 1, 2, 3 and 4 are used to compare the differences between different experimental groups

calorie-matched continuous CR improved insulin sensitivity. Overall, these results suggest that intermittent FMD and continuous $\mathrm{CR}$ are almost equally effective in improving the glycemic control in the T1D mice, except for that the 2-5 regime of intermittent CR performed better than continuous CR in improving insulin sensitivity and the 59 regime of intermittent FMD performed better than continuous $C R$ in improving glucose tolerance.

We also directly compared the glycemic control data between the 2-5 and 5-9 protocols in both STZ-treated and $d b / d b$ mice (shown in Additional file 1: Figure S6). For glycemic control, both intermittent FMD and continuous CR had the same effect to significantly lower the fasting blood glucose level in STZ-treated mice (Additional file 1: Figure S6A). However, in $d b / d b$ mice, intermittent FMD protocols were better than their matched continuous $\mathrm{CR}$ groups (Additional file 1: Figure S6B). We next compared different parameters in $d b / d b$ mice (Additional file 1: Fig. S7). Only continuous CR matching 5-9 regime gave rise to significant reduction of body weight (Additional file 1: Figure S7A). Consistent with the result of the fasting glucose level (Additional file 1: Figure S7B), both GTT and ITT were significantly better in intermittent FMD groups than their matched continuous CR groups (Additional file 1: Figure S7C and D). These comparisons, therefore, indicated that intermittent FMD performs better than continuous $\mathrm{CR}$ in improving glycemic control, glucose tolerance and insulin sensitivity in $d b / d b$ mice.

\section{Immunofluorescent analysis of the pancreatic islets}

Endocrine cells from pancreatic islets include $\alpha$ cells, $\beta$ cells, $\delta$ cells, and pancreatic polypeptide (PP) cells, which produce glucagon, insulin, somatostatin, and pancreatic polypeptide respectively $[14,15]$. The $\alpha$ cells commonly form a continuous mantle around the $\beta$ cells in rodents [16]. We performed immunofluorescent staining with the pancreas sections of the mice to analyze both insulin-secreting $\beta$ cells and glucagon-secreting $\alpha$ cells. In normal C57BL/ 6 mice, the majority of $\beta$ cells were localized in the middle of the islets, surrounded by $\alpha$ cells in the peripheral zone (Fig. 6a and Additional file 1: Figure S3A). Most of the islets were markedly disrupted in both $d b / d b$ and T1D mice with very few $\beta$ and $\alpha$ cells present (Fig. 6b and Additional file 1: Figure S3B and C). Both regimes of intermittent FMD profoundly restored the islet structures. Most importantly, both 2-5 and 5-9 protocols of intermittent FMD could significantly increase the number of $\beta$ cells in both $d b / d b$ and STZtreated mice (Fig. 6, Additional file 1: Figures S4 and S5). However, the distribution pattern of insulin-positive cells and glucagon-positive cells were not identical to the normal mice (Fig. 6, Additional file 1: Figures S4 and S5). In detail, some glucagon-positive cells were localized in the middle area in the islets instead of peripheral distribution in normal islets after diet intervention. Continuous CR could partially recover the insulin-positive cells in the islets, but not to the same degree as the intermittent FMD in the majority of the experiments (Fig. 6, Additional file 1: Figures S4 and S5).

\section{Discussion}

This study analyzed the intervention activity of different protocols of intermittent calorie restriction. Our original protocol used a 7-7 regime in which the mice were given FMD containing $1 / 3 \mathrm{cal}$ intake of the control mice 


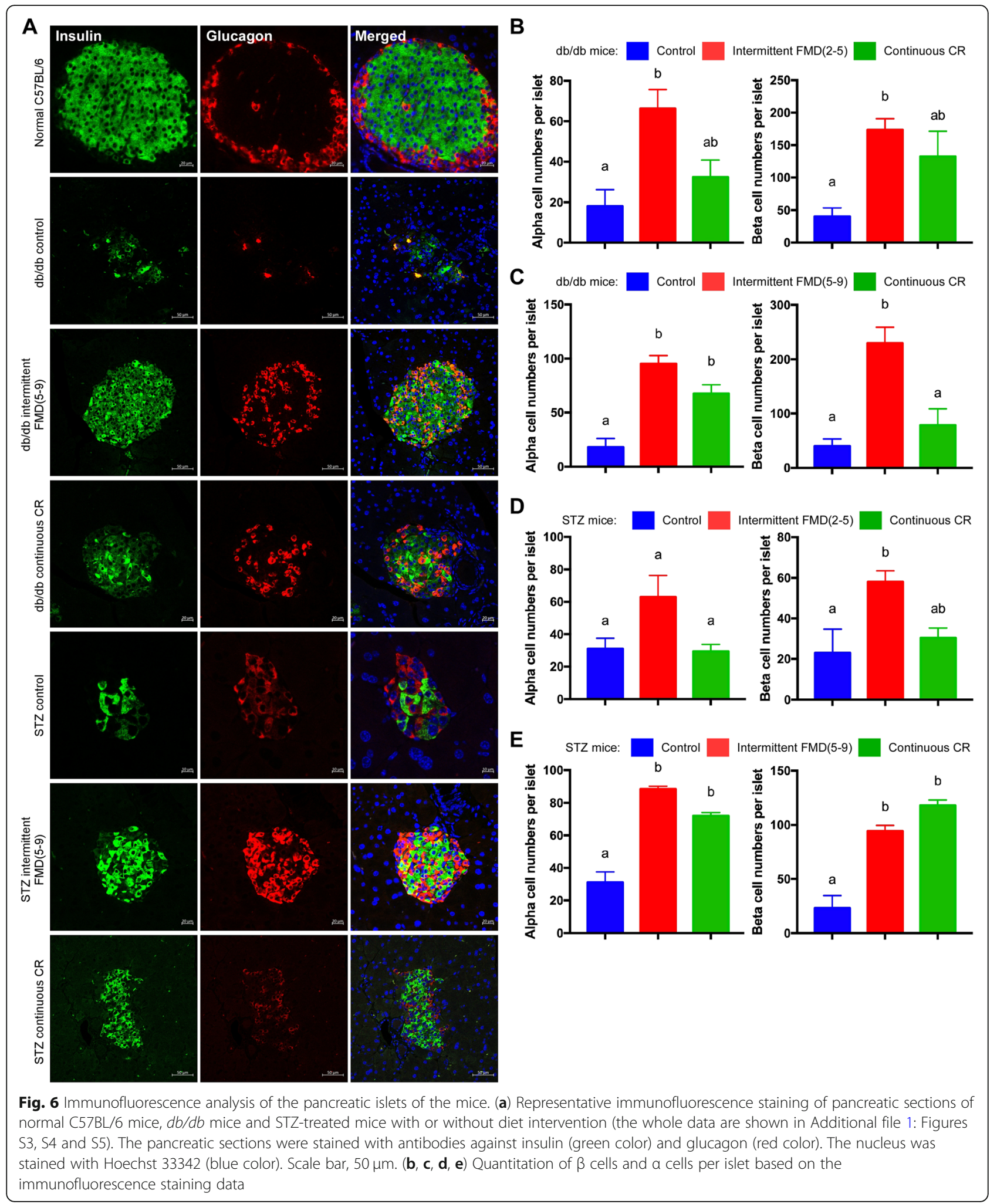

for 7 days, followed by 7 days' free eating [12]. In this study, we tried both 2-5 and 5-9 protocols in $d b / d b$ and STZ-treated mice. The purpose of trying the intervention activity of different fasting protocols was to pave the way for future application in humans. We expect that shorter term of calorie restriction would be easier to be accepted by diabetic patients. Therefore, it is ideal to discover an effective intervention protocol that has 
the shortest period of intermittent fasting. It is noteworthy that the blood glucose level fluctuated in the first week in intermittent FMD groups (Figs. 1, 2, 3 and 4). This is likely due to the fact that these mice hadn't been adapted to the new eating pattern.

One of the key findings of this study is that while both intermittent $\mathrm{CR}$ and continuous CR could significantly improve glycemic control, intermittent $\mathrm{CR}$ performed better than continuous CR in $d b / d b$ mice (Figs. 1, 2 and 5). To our knowledge, this is the first study that directly compares the interventional activity of intermittent CR vs continuous $C R$ within the same study in the mice. Intriguingly, it was found that intermittent fasting is better than continuous $\mathrm{CR}$ in improving longevity in C. elegans [17], consistent with our finding in certain degree. We postulate that intermittent $\mathrm{CR}$ accommodates better than continuous $\mathrm{CR}$ to the adaptation of most animals including humans during over millions of years of evolution. In nature, most animals commonly experience periodic long-term fasting interweaved with short-term feast. Accordingly, the metabolic machinery of most animals has adapted to such periodic dietary pattern. Only in recent years has this periodic dietary pattern in humans been replaced by an almost continuous eating pattern (such as three meals a day) due to the advances of agriculture and technology. Based on the viewpoint of evolution, it can be argued that periodic or intermittent CR should perform better than continuous CR to improve metabolic health and possibly aging in humans when over-nutrition has become a worldwide problem. As nicely summarized by Di Francesco and colleagues, periodic calorie restriction can mediate "periodic shifts of metabolic fuel sources, promotion of repair mechanisms, and the optimization of energy utilization for cellular and organismal health" [18]. With this idea in mind, research is urgently needed in human subjects to directly compare the activities of intermittent CR vs continuous $\mathrm{CR}$ in improving metabolic health and reducing risk factors of metabolic disorders.

Another interesting finding in our study is that loss of body weight is not necessarily associated with improvement of glycemic control in the mice. In our study, intermittent $C R$ did not significantly reduce the body weight of $d b / d b$ mice (Figs. 1, 2 and 5). However, continuous $C R$ significantly decreased the body weight of the $d b / d b$ mice when using 5-9 regime (Figs. 2 and 5). This discrepancy is likely caused by the fact that the mice using FMD might eat more when having free access to the food after stopping FMD (Additional file 1: Figure S1), while the food intake was constantly limited to the mice with continuous CR. However, improvement of glycemic control occurred in the mice with intermittent CR, without significant loss of body weight. Therefore, our observations indicate that intermittent CR has an independent effect to improve metabolic health in addition to the commonly believed beneficial effect of body weight reduction.

Our studies with the pancreatic islets provided additional evidence that intermittent $\mathrm{CR}$ might perform better than continuous $C R$ in restoring the disrupted $\beta$ cells in diabetic mice (Fig. 6). Based on the staining of insulin- and glucagon-positive cells in the islets, it appears that the 5-9 regime of intermittent CR performed the best in increasing the insulin-positive cells in the islets, better than the calorie-matched continuous CR (Fig. 6). Notably, there were many glucagon-positive cells in the central area of the islets in $d b / d b$ mice and STZ-treated mice (Fig. 6 and Additional file 1: Figure S3), indicating a likely $\beta$-to- $\alpha$ transition in diabetic state. Interestingly, many cells in the islets were positive for both insulin and glucagon in $d b / d b$ mice after intermittent FMD application (Fig. 6 and Additional file 1: Figure S4). This observation indicates there might be interconversion of $\alpha$-to- $\beta$ in the islets, likely explaining the interventional effect of intermittent CR in improving glycemic control in the diabetic mice. Nevertheless, our results indicated that intermittent CR could significantly restore the disrupted $\beta$ cells in the diabetic mice.

Besides, other mechanisms might contribute to the interventional activity of intermittent CR. We analyzed the activity of insulin signaling in the skeletal muscle and liver as the ITT results (Figs. 1, 2, 3 and 4) indicated that insulin sensitivity was elevated by intermittent FMD. C57BL/6 mice were treated with STZ and then administrated with or with intermittent FMD for three weeks. The mice were fasted for $5 \mathrm{~h}$ in the morning and then injected with or without insulin. We analyzed the phosphorylation of AKT in both skeletal muscle and liver tissues. We found that intermittent FMD could significantly enhance insulin-stimulated AKT phosphorylation in these tissues (Additional file 1: Figure S8), indicating that insulin signaling is elevated by intermittent FMD.

In addition, our study revealed that intermittent $\mathrm{CR}$ was able to improve the glycemic control in STZ-treated mice, similar to previous reports $[11,12]$. However, intermittent $\mathrm{CR}$ was almost as equally effective as continuous $\mathrm{CR}$ in the improvement of fasting glucose and insulin sensitivity (Figs. 3, 4 and 5). The discrepancy of intermittent $C R$ in the improvement of glycemic control between $d b / d b$ mice (in which it is better than continuous CR) and STZ-treated mice is likely caused by the difference in the pathogenesis of type 2 diabetes vs type 1 diabetes. In type 2 diabetes, both insulin resistance and $\beta$ cells dysfunction contribute to the destruction of glucose homeostasis. However, the damage of $\beta$ cells is the primary cause of type 1 diabetes. It will be of interest in the future to compare intermittent $\mathrm{CR}$ vs continuous $\mathrm{CR}$ 
using the NOD mouse model that mimics type 1 diabetes in humans better than STZ treatment. Nevertheless, our study has provided evidence that intermittent CR with FMD stands out as a promising strategy for the intervention of diabetes mellitus.

\section{Conclusions}

Our study indicated that both intermittent CR and continuous CR can lower fasting blood glucose level in the diabetic mice, while intermittent $\mathrm{CR}$ is better than the latter in improving glucose homeostasis in $d b / d b$ mice. Considering the effectiveness of intermittent $C R$ in the intervention of diabetes in mice, this study would pave the way for carrying out similar studies in humans to control the progression of diabetes mellitus.

\section{Additional file}

\section{Additional file 1: Figure S1. Food intake of the mice. Figure S2.} Comparison of 2-5 regime of intermittent fasting using normal chow with intermittent FMD and matched continuous CR in $\mathrm{db} / \mathrm{db}$ mice. Figure S3. Fluorescent staining of pancreatic islets in control mice. Figure S4. Fluorescent staining of pancreatic islets in $\mathrm{db} / \mathrm{db}$ mice after diet intervention. Figure S5. Fluorescent staining of pancreatic islets in STZ-treated mice after diet intervention. Figure S6. Comparison of glycemic control between intermittent FMD and continuous CR in $\mathrm{db} / \mathrm{db}$ and STZ-treated mice. Figure S7. Comparison between intermittent FMD and continuous $C R$ in $\mathrm{db} / \mathrm{db}$ mice. Figure $\mathbf{S 8}$. Intermittent FMD enhances insulin signaling in the skeletal muscle and liver of the mice. (PDF $1400 \mathrm{~kb})$

\section{Acknowledgments}

We like to thank Susie Chen at University of Pittsburgh for editorial assistance.

\section{Authors' contributions}

YC conceptualized the project. SW and YC designed the study, analyzed the data and wrote the paper. S.W. performed the experiments. JZ, MB, CL, and LZ provided technical assistance. All authors have read and approved the manuscript.

\section{Funding}

This study was supported by research grants from the National Natural Science Foundation of China (31630036 to Y.C.), the Ministry of Science and Technology of China (2016YFA0500103 to Y.C.), and the Chinese Academy of Sciences (XDA12010102, QYZDJ-SSW-SMC008, ZDRW-ZS-2016-8, and CAS Interdisciplinary Innovation Team to Y.C.).

\section{Availability of data and materials}

All data generated or analysed during this study are included in this published article and its Additional file.

\section{Ethics approval and consent to participate}

Not applicable.

\section{Consent for publication}

Not applicable.

\section{Competing interests}

The authors declare that they have no competing interests.
Received: 15 May 2019 Accepted: 16 August 2019

Published online: 28 August 2019

\section{References}

1. Omodei D, Fontana L. Calorie restriction and prevention of age-associated chronic disease. FEBS Lett. 2011;585:1537-42.

2. Longo VD, Mattson MP. Fasting: molecular mechanisms and clinical applications. Cell Metab. 2014;19:181-92.

3. Golbidi S, Daiber A, Korac B, Li H, Essop MF, Laher I. Health benefits of fasting and caloric restriction. Curr Diab Rep. 2017;17:123.

4. Mattson MP, Longo VD, Harvie M. Impact of intermittent fasting on health and disease processes. Ageing Res Rev. 2017;39:46-58.

5. Anton SD, Moehl K, Donahoo WT, Marosi K, Lee SA, Mainous AG 3rd, Leeuwenburgh C, Mattson MP. Flipping the metabolic switch: understanding and applying the health benefits of fasting. Obesity (Silver Spring). 2018;26:254-68.

6. Wing RR, Blair EH, Bononi P, Marcus MD, Watanabe R, Bergman RN. Caloric restriction per se is a significant factor in improvements in glycemic control and insulin sensitivity during weight loss in obese NIDDM patients. Diabetes Care. 1994;17:30-6.

7. Brandhorst S, Choi IY, Wei M, Cheng CW, Sedrakyan S, Navarrete G, Dubeau $L$, Yap LP, Park R, Vinciguerra $M$, et al. A periodic diet that mimics fasting promotes multi-system regeneration, enhanced cognitive performance, and Healthspan. Cell Metab. 2015;22:86-99.

8. Choi IY, Piccio L, Childress P, Bollman B, Ghosh A, Brandhorst S, Suarez J, Michalsen A, Cross AH, Morgan TE, et al. A diet mimicking fasting promotes regeneration and reduces autoimmunity and multiple sclerosis symptoms. Cell Rep. 2016;15:2136-46.

9. Di Biase S, Lee C, Brandhorst S, Manes B, Buono R, Cheng CW, Cacciottolo M, Martin-Montalvo A, de Cabo R, Wei M, et al. Fasting-mimicking diet reduces HO-1 to promote T cell-mediated tumor cytotoxicity. Cancer Cell. 2016;30:136-46.

10. Wei M, Brandhorst S, Shelehchi M, Mirzaei H, Cheng CW, Budniak J, Groshen S, Mack WJ, Guen E, Di Biase S, et al. Fasting-mimicking diet and markers/ risk factors for aging, diabetes, cancer, and cardiovascular disease. Sci Trans Med. 2017;9.

11. Cheng CW, Villani V, Buono R, Wei M, Kumar S, Yilmaz OH, Cohen P, Sneddon JB, Perin L, Longo VD. Fasting-mimicking diet promotes Ngn3driven beta-cell regeneration to reverse diabetes. Cell. 2017;168:775-788 e712.

12. Wei S, Han R, Zhao J, Wang S, Huang M, Wang Y, Chen Y. Intermittent administration of a fasting-mimicking diet intervenes in diabetes progression, restores beta cells and reconstructs gut microbiota in mice. Nutr Metab (Lond). 2018:15:80.

13. Feng L, Xie X, Ding Q, Luo X, He J, Fan F, Liu W, Wang Z, Chen Y. Spatial regulation of Raf kinase signaling by RKTG. Proc Natl Acad Sci U S A. 2007; 104:14348-53.

14. Shih HP, Wang A, Sander M. Pancreas organogenesis: from lineage determination to morphogenesis. Annu Rev Cell Dev Biol. 2013;29:81-105.

15. Larsen $\mathrm{HL}$, Grapin-Botton A. The molecular and morphogenetic basis of pancreas organogenesis. Semin Cell Dev Biol. 2017;66:51-68.

16. Steiner DJ, Kim A, Miller K, Hara M. Pancreatic islet plasticity: interspecies comparison of islet architecture and composition. Islets. 2010;2:135-45.

17. Honjoh S, Yamamoto T, Uno M, Nishida E. Signalling through RHEB-1 mediates intermittent fasting-induced longevity in C. elegans. Nature. 2009; 457:726-30

18. Di Francesco A, Di Germanio C, Bernier M, de Cabo R. A time to fast. Science. 2018;362:770-5.

\section{Publisher's Note}

Springer Nature remains neutral with regard to jurisdictional claims in published maps and institutional affiliations. 